\title{
The relationship between 25 -hydroxyvitamin $D$ concentration in early pregnancy and pregnancy outcomes in a large, prospective cohort
}

\author{
Veronica T. Boyle ${ }^{1 *}$, Eric B. Thorstensen ${ }^{1}$, David Mourath ${ }^{2}$, M. Beatrix Jones ${ }^{3}$, Lesley M. E. McCowan ${ }^{4}$, \\ Louise C. Kenny ${ }^{5}$ and Philip N. Baker ${ }^{1,6}$ on behalf of the SCOPE Consortium \\ ${ }^{1}$ Gravida: National Centre for Growth and Development, The Liggins Institute, The University of Auckland, Auckland 1023, \\ New Zealand \\ ${ }^{2}$ Medical Program, Linköping University, Linköping, SE-581 83, Sweden \\ ${ }^{3}$ Institute of Natural and Mathematical Sciences, Massey University, Auckland 0632, New Zealand \\ ${ }^{4}$ The Department of Obstetrics and Gynaecology, South Auckland Clinical School, The University of Auckland, Auckland \\ 1023, New Zealand \\ ${ }^{5}$ The Irish Centre for Fetal and Neonatal Translational Research (INFANT) and the Department of Obstetrics and Gynaecology, \\ University College Cork, Cork, Ireland \\ ${ }^{6}$ The College of Medicine, Biological Sciences and Psychology, University of Leicester, Leicester LE1 7RH, UK \\ (Submitted 23 March 2016 - Final revision received 30 June 2016 - Accepted 8 August 2016)
}

\section{Abstract}

Vitamin D insufficiency and deficiency have been associated with an increased risk of adverse pregnancy outcomes. Controversy remains as findings have been inconsistent between disparate populations. The aim of this study was to investigate the relationship between vitamin $\mathrm{D}$ status and pregnancy outcomes in a large, prospective pregnancy cohort. 25-Hydroxyvitamin D concentration was analysed in serum samples collected at 15 weeks of gestation from 1710 New Zealand women participating in a large, observational study. Associations between vitamin D status and pre-eclampsia, preterm birth, small for gestational age (SGA) and gestational diabetes were investigated. The mean 25 -hydroxyvitamin D concentration was $72.9 \mathrm{nmol} / \mathrm{l}$. In all, $23 \%$ had 25 -hydroxyvitamin D concentrations $<50 \mathrm{nmol} / 1$, and $5 \%$ of participants had concentrations $<25 \mathrm{nmol} / \mathrm{l}$. Women with 25-hydroxyvitamin D concentrations $<75 \mathrm{nmol} / 1$ at 15 weeks of gestation were more likely to develop gestational diabetes mellitus than those with concentrations $>75 \mathrm{nmol} / 1$ (OR $2 \cdot 3 ; 95 \%$ CI 1.1, 5.1). However, this effect was not significant when adjustments were made for BMI and ethnicity (OR 1·8; $95 \%$ CI 0·8, 4.2). 25-Hydroxyvitamin D concentration at 15 weeks was not associated with development of pre-eclampsia, spontaneous preterm birth or SGA infants. Pregnancy complications were low in this largely vitamin D-replete population.

\section{Key words: Vitamin D: Gestational diabetes mellitus: Pre-eclampsia}

Much progress has been made in the screening, diagnosis and management of important pregnancy disorders, but little progress has been made in the prevention of such disorders. Preeclampsia remains the second most common cause of maternal death worldwide ${ }^{(1)}$. Prematurity, mostly due to spontaneous preterm birth, is the leading cause of death among infants worldwide $^{(2)}$. This is followed by growth restriction in term infants $^{(3)}$. Furthermore, infants born preterm or with low birth weight have an increased risk of CVD and metabolic diseases later in life, the leading cause of death in adulthood ${ }^{(4)}$. The incidence of gestational diabetes mellitus (GDM) is increasing rapidly worldwide ${ }^{(5)}$ and is associated with lifelong risks of metabolic disease in both mother and baby ${ }^{(6,7)}$.

An association between low vitamin D and adverse pregnancy outcomes was first identified in the early $2000 \mathrm{~s}^{(8)}$; consequently, vitamin $\mathrm{D}$ has been postulated as a possible intervention strategy to reduce pregnancy complications. A recent meta-analysis found that low vitamin $\mathrm{D}$, defined variably by the authors of included studies, was associated with an increased likelihood of pre-eclampsia (OR 1.79; 95\% CI 1.25, 2.58), small-for-gestational age (SGA) babies (OR 1.85; 95\% CI $1.52,2 \cdot 26)$ and GDM (OR 1.49; $95 \%$ CI 1.18, 1.89) $)^{(9)}$. Preterm birth was not included as an outcome. However, most included studies did not correct for important confounders, and those that did were small. In addition, vitamin D deficiency was variably defined between 35 and $80 \mathrm{nmol} / 1$ or analysed in late pregnancy ${ }^{(9)}$.

Late pregnancy may not be the optimum time to measure vitamin $\mathrm{D}$, as it is believed that the origins of most late pregnancy disorders are in early pregnancy. Moreover, the expressions of both CYP25B1 (the vitamin D activating enzyme) and vitamin D

Abbreviations: GDM, gestational diabetes mellitus; NZ, New Zealand; SCOPE, Screening for Pregnancy Endpoints study; SGA, small for gestational age. 
nuclear receptor ${ }^{(10)}$ are highest during the first trimester. In the current study, factors that may alter the development of pregnancy conditions were investigated at the beginning of the second trimester - a time when critical vascular development of the placenta occurs ${ }^{(11)}$.

The aim of this study was to investigate the relationship between early pregnancy 25-hydroxyvitamin D concentration and subsequent development of pre-eclampsia, spontaneous preterm birth, SGA and GDM in a large, well-phenotyped, prospective cohort.

\section{Methods}

\section{Participants}

Samples were collected from Auckland participants recruited to the Screening for Pregnancy Endpoints study (SCOPE) between 2005 and 2008. SCOPE is an international, prospective, pregnancy study of nulliparous women, which aims to identify early pregnancy predictors of late pregnancy complications ${ }^{(12)}$. The study was approved by the Auckland University Ethics Committee (AKX/02/00/364). The trial was registered with the Australia New Zealand clinical trial registry (ACTRN 12607000551493).

Non-fasting serum samples were collected at 15 weeks of gestation and stored at $-80^{\circ} \mathrm{C}$. At the time of sample collection, participants were asked whether they took multivitamins, and the brand used was recorded. Data on socio-economic status ${ }^{(13)}$ were recorded, and participants were asked whether they smoked. Participants were also asked to rate their physical activity (over the previous 3 months) as vigorous, moderate, recreational walking or no physical activity, and the frequency at which they engaged in physical activity was recorded. Data were entered into an Internet-accessed, password-protected, centralised database with complete audit train (MedSciNet ${ }^{\mathrm{AB}}$ ).

Pre-eclampsia was diagnosed according to the International Society for the Study of Hypertension in Pregnancy criteria ${ }^{(14)}$ as gestational hypertension (systolic blood pressure of $\geq 140 \mathrm{mmHg}$ and/or diastolic blood pressure of $\geq 90 \mathrm{mmHg}$ on at least two occasions) with proteinuria or any multisystem complication of pre-eclampsia(15). SGA was defined as weight below the 10th customised birth weight centile ${ }^{(16)}$, and spontaneous preterm birth was defined as delivery before 37 weeks of gestation. Women were screened for GDM between 24 and 28 weeks of gestation with a non-fasting 50-g polycose challenge in community laboratories, according to the Auckland District Health Board Guidelines. Those with a positive polycose test (defined as $1 \mathrm{~h}$ post-challenge glucose of $\geq 7 \cdot 8 \mathrm{mmol} / \mathrm{l}$ ) were further tested with a fasting 75-g oral glucose tolerance test (OGTT). A total of forty-four women considered to be at high risk of GDM underwent an OGTT without a polycose test. GDM was diagnosed if fasting glucose was $\geq 5.5 \mathrm{nmol} / 1$ or if the 2-h post-glucose challenge showed values $\geq 9 \cdot 0 \mathrm{mmol} / \mathrm{l}$.

\section{Sample analysis}

Serum samples were prepared using Phree $^{\mathrm{TM}}$ phospholipid removal cartridges (Phenomenex) after addition of the internal standard deuterated 25-hydroxyvitamin $\mathrm{D}_{3}$ (Isosciences). Separation of 25-hydroxyvitamin $\mathrm{D}_{3}$ and 3-epi-25-hydroxyvitamin $\mathrm{D}_{3}$ was achieved with a Kinetex ${ }^{\mathrm{TM}}$ pentaflurophenyl $100 \times 3-\mathrm{mm} 2 \cdot 6-$ $\mu \mathrm{m}$ HPLC column (Phenomenex). 25-Hydroxyvitamin $\mathrm{D}_{3}, \mathrm{c} 3-$ epi 25-hydroxyvitamin $\mathrm{D}_{3}$ and 25-hydroxvitamin $\mathrm{D}_{2}$ were analysed by liquid chromatography tandem MS (Finnigan TSQ Quantum Ultra ${ }^{\mathrm{TM}}$ triple quadrupole mass spectrometer; Thermo Electron Corporation). Total 25-hydroxyvitamin D was calculated by the sum of these three compounds. The intra- and inter-assay $\mathrm{CV}$ were 7.3 and $4.9 \%$ for 25 -hydroxyvitamin $\mathrm{D}_{3}$ and 8.2 and $5.8 \%$ for 25 -hydroxyvitamin $\mathrm{D}_{2}$, respectively. Quality control was ensured through participation in the Vitamin D External Quality Assessment Scheme DEQAS programme operating out of Charing Cross Hospital, London ${ }^{(17)}$, and the use of commercially available control materials of known vitamin D concentration. Vitamin D binding protein (VDBP) was analysed using an ELISA from R\&D systems.

\section{Statistical analysis}

All statistical analyses were performed in SPSS (IBM) version 21. Normality was tested by assessing the histogram and tests for skewness and kyphosis. Continuous variables are reported as mean values and standard deviations. $\chi^{2}$ Tests were performed for categorical variables and the $t$ test for continuous variables. Fisher's exact test was performed when $\chi^{2}$ was not appropriate. The dates of sample collection were categorised into seasons as follows: summer, December to February; autumn, March to May; winter, June to August; and spring, September to November. Logistic regression analyses were performed to determine whether 25-hydroxyvitamin D concentration was associated with the development of GDM, pre-eclampsia, SGA and spontaneous preterm birth. Only participants screened for GDM were included in the analysis for GDM. Multiple logistic regression with backwards selection using the likelihood ratio criteria was used to incorporate known risk factors for adverse pregnancy outcomes, including age, BMI, ethnicity, smoking status and socio-economic status. The analysis was performed with 25 -hydroxyvitamin D as a continuous variable and vitamin $D$ level as a categorical variable. Vitamin D categorical variables were created with cut-offs at 25,50 and $75 \mathrm{nmol} / \mathrm{l}$. These cut-offs were based on the Institute of Medicine definition of deficiency $(<50 \mathrm{nmol} / \mathrm{l})^{(18)}$ and the Endocrine Society clinical practice guidelines definition of insufficiency $(<75 \mathrm{nmol} / \mathrm{l})$ and severe deficiency $(<25 \mathrm{nmol} / \mathrm{l})^{(19)}$. HosmerLemeshow goodness of fit was performed to test the adequacy of the model (a value of 1 indicates perfect correspondence between the model-predicted and observed risks).

\section{Results}

A total of 2065 women were recruited to the SCOPE study in Auckland. Of those women, 355 did not have 15-week serum or plasma samples for analysis. In total, 1710 women were included in the final analysis. Of those, 166 participants were not screened for GDM, and 1544 women were included in the final analysis for that outcome. Among them, thirty-two (2.1\%) women were diagnosed with GDM, seventy-three (4.2\%) women were diagnosed with pre-eclampsia, seventy ( $4 \cdot 1 \%)$ women spontaneously delivered preterm infants and 170 (9.9\%) women delivered SGA infants (Fig. 1). The majority of women were of New Zealand 


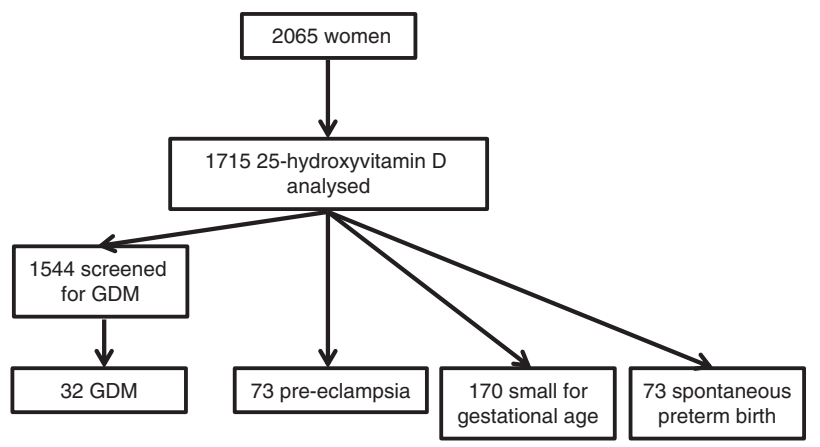

Fig. 1. Cohort flow diagram of Auckland SCOPE participants from recruitment at 15 weeks' gestation.

(NZ) European ethnicity (83.8\%). The remaining ethnic groups were Asian ( $n 91,5.3 \%$ ), Maori ( $n 57,3.3 \%$ ), Indian ( $n 65,3.8 \%$ ), Pacific Islanders ( $n$ 34, $2 \cdot 0 \%)$ and African ( $n$ 30, 1.8\%) (combined as 'other ethnicities' in Table 1).

Overall, $55 \%$ of women took multivitamin supplements containing vitamin $\mathrm{D}$; one brand contained vitamin $\mathrm{D}_{2}$ and was taken by twenty women. The dose of vitamin $\mathrm{D}$ supplement ranged from 5 to $20 \mu \mathrm{g} / \mathrm{d}$. Women who took multivitamin supplements containing vitamin D had significantly higher 25-hydroxyvitamin $\mathrm{D}$ concentrations than those who did not (93.6 (sD 29.6) $v .75 .8(\mathrm{sD} 32.6) \mathrm{nmol} / \mathrm{l} ; P<0.001)$. The proportion of women who took multivitamin supplements containing vitamin D was lower among those who developed GDM, although this was not statistically significant (38v. $55 \% ; P=0 \cdot 4)$.

Concentrations of 25-hydroxyvitamin D were approximately normally distributed (skewness -0.03, SE 0.05; kurtosis $-0 \cdot 3$, SE $0 \cdot 1)$. Just over half of the sample (53\%) had 25-hydroxyvitmamin D concentrations $<75 \mathrm{nmol} / 1,22 \cdot 7 \%$ had concentrations $<50 \mathrm{nmol} / 1$ and $4.4 \%$ had concentrations $<25 \mathrm{nmol} / \mathrm{l}$. There was a significant difference in the mean BMI between women who developed GDM and pre-eclampsia and women who did not $(P=0.001)$ (Table 1$)$. The prevalence of GDM and pre-eclampsia varied significantly depending on ethnicity ( $P=0.02$ and 0.05 , respectively), with Indian women having the highest prevalence of GDM and NZ European women the lowest. Pacific Island women had the highest prevalence of pre-eclampsia. The mean total serum 25-hydroxyvitamin D concentration was lower in those who developed GDM than in those who did not $(61.6$ (SD 23.9) nmol/l as compared with 72.9 (sD 27.0$) \mathrm{nmol} / \mathrm{l} ; \quad P=0.03$ ) but not lower in women who developed pre-eclampsia $(68 \cdot 1 \quad(\mathrm{SD} 27 \cdot 8) \mathrm{nmol} / \mathrm{l})$ or delivered preterm $(73.7$ (SD 27.5$) \mathrm{nmol} / \mathrm{l})$ or SGA infants $(70 \cdot 6$ (sD 29.0) nmol/l) (Table 1). Of women who developed GDM, $72 \%$ had a concentration $<75 \mathrm{nmol} / 1$ and $22 \%$ had a concentration $<50 \mathrm{nmol} / \mathrm{l}$. Women of European decent were the largest ethnic group; there was no significant difference in mean total 25-hydroxyvitamin D in those who developed GDM and in those who did not $(70 \cdot 6 v .75 .9 \mathrm{nmol} / \mathrm{l} ; P=0.35)$.

There was an increased likelihood of developing GDM if total 25-hydroxyvitamin D was $<75 \mathrm{nmol} / \mathrm{l}$ (OR 2·3; $95 \%$ CI 1·1, 5·1). However, after adjustment for BMI and ethnicity, this difference was not significant (adjusted OR 1.8; 95\% CI 0.8, 4.2). There was no increased likelihood of developing any of the adverse pregnancy outcomes at concentrations $<50$ and $<25 \mathrm{nmol} / 1$ (Table 2).

On logistic regression, 25-hydroxyvitamin D concentration as a continuous variable was associated with an increased likelihood of developing GDM with a $1 \%$ increase in risk for every $1 \mathrm{nmol} / 1$ reduction in 25-hydroxyvitamin D (OR 1.01; $95 \% \mathrm{CI}$ $1 \cdot 01,1 \cdot 03 ; P=0.03$ ) (Table 2). Following inclusion of BMI and ethnicity in the model, the relationship between 25-hydroxyvitamin D concentration and GDM was no longer significant. Using the International Association of Diabetes in Pregnancy criteria for the diagnosis of GDM increased the number of GDM cases ( $n$ 48) but did not increase the significance of the relationship with 25-hydroxyvitamin D.

In a logistic regression model including vitamin $\mathrm{D}$ and season, vitamin $\mathrm{D}$ was not a significant contributor. After adjustment for BMI and ethnicity, season remained a significant risk factor for GDM (adjusted OR 4.2; $95 \%$ CI 1.3, 13.0; $P=0.04)$. Season was also a significant predictor of developing pre-eclampsia (adjusted OR 2.2; 95\% CI 1.0, 4.5; $P=0.04$ ) and delivering SGA infants (adjusted OR 2.1; $95 \%$ CI 1.3, 3.5; $P=0.003)$. After adjustment for BMI and ethnicity, exercise $(P=0 \cdot 1)$, age $(P=0 \cdot 7)$, socio-economic status $(P=0 \cdot 8)$ and smoking status $(P=0 \cdot 2)$ were not statistically associated with GDM and are not included in the model shown in Table 2.

VDBP was analysed, and free 25-hydroxyvitamin D was calculated for a subgroup of participants (32 GDM with 4 BMI- and ethnicity-matched controls). VDBP concentration was significantly correlated with 25-hydroxyvitamin D concentration $(P=0 \cdot 03)$. No correlation between calculated free 25-hydroxyvitamin D and GDM was found.

\section{Discussion}

In this large, well-phenotyped cohort, we found that 25-hydroxyvitmain D concentration was not lower at 15 weeks of gestation in women who later developed pre-eclampsia, spontaneous preterm birth or who had SGA infants. Concentrations were lower in women who developed GDM but did not predict GDM when adjusted for BMI and ethnicity.

This cohort differs from most other pregnancy cohorts in that it is relatively vitamin D replete. Although $23 \%$ of Auckland SCOPE participants were vitamin D deficient (25-hydroxyvitamin $\mathrm{D}<50 \mathrm{nmol} / \mathrm{l}$ ), half to more than $90 \%$ of pregnant women have been found to be deficient in previous studies $^{(20-22)}$. The SCOPE Auckland cohort may be more vitamin $\mathrm{D}$ replete in comparison with the obstetric Auckland population and the wider New Zealand population. Auckland is in the northern region of New Zealand where it is estimated that $24 \mathrm{~min}$ of hand and face exposure to the sun daily is adequate. This is not the case for much of New Zealand's geographical distribution ${ }^{(23)}$. NZ European women had the highest 25-hydroxyvitamin D concentrations by ethnicity, and although they represented $83.8 \%$ of Auckland Scope, at the time of sample collection, they represented $49.0 \%$ of women delivering at Auckland City Hospital ${ }^{(24)}$, and according to the most recent National Women's report they represented $44 \%$ of women delivering at Auckland city hospital ${ }^{(25)}$. 
Table 1. Maternal characteristics, 25-hydroxyvitamin D concentration at 15 weeks of gestation and pregnancy outcomes (Numbers and percentages; mean values and standard deviations)

\begin{tabular}{|c|c|c|c|c|c|c|c|c|c|c|}
\hline & \multicolumn{2}{|c|}{ All $n 1710$} & \multicolumn{2}{|c|}{ Pre-eclampsia $n 72$} & \multicolumn{2}{|c|}{ sPTB $n 73$} & \multicolumn{2}{|c|}{ SGA $n 170$} & \multicolumn{2}{|c|}{ GDM $n 32$} \\
\hline & $n$ & $\%$ & $n$ & $\%$ & $n$ & $\%$ & $n$ & $\%$ & $n$ & $\%$ \\
\hline \multicolumn{11}{|l|}{ Age (years) } \\
\hline Mean & \multicolumn{2}{|c|}{$30 \cdot 3$} & \multicolumn{2}{|c|}{$30 \cdot 0$} & \multicolumn{2}{|c|}{$30 \cdot 8$} & \multicolumn{2}{|c|}{$31 \cdot 1$} & \multicolumn{2}{|c|}{$30 \cdot 8$} \\
\hline SD & \multirow{2}{*}{\multicolumn{2}{|c|}{4.7}} & \multicolumn{2}{|c|}{5.0} & & & \multicolumn{2}{|c|}{4.8} & \multicolumn{2}{|c|}{$5 \cdot 1$} \\
\hline \multicolumn{10}{|l|}{ BMI $\left(\mathrm{kg} / \mathrm{m}^{2}\right)$} & \\
\hline Mean & \multirow{3}{*}{\multicolumn{2}{|c|}{$\begin{array}{r}24.8 \\
4.2\end{array}$}} & \multicolumn{2}{|c|}{$26 \cdot 9$} & & & \multicolumn{2}{|c|}{$25 \cdot 4$} & \multicolumn{2}{|c|}{$27 \cdot 4$} \\
\hline SD & & & \multirow{2}{*}{\multicolumn{2}{|c|}{$\begin{array}{c}4.8 \\
P=0.05\end{array}$}} & & & \multirow{2}{*}{\multicolumn{2}{|c|}{$\begin{array}{c}5.1 \\
P=0.18\end{array}$}} & \multirow{2}{*}{\multicolumn{2}{|c|}{$\begin{array}{c}5.2 \\
P=0.02\end{array}$}} \\
\hline Ethnicity & & & & & & & & & & \\
\hline NZ European & 1433 & 83.8 & 54 & 3.8 & 67 & 4.7 & 137 & 9.6 & 21 & 1.6 \\
\hline Other ethnicities & 277 & $16 \cdot 2$ & 18 & 6.5 & 6 & $2 \cdot 1$ & 33 & 11.9 & 11 & $4 . c$ \\
\hline Season at sample collection & & & \multicolumn{2}{|c|}{$P=0.01$} & \multicolumn{2}{|c|}{$P=0.9$} & \multicolumn{2}{|c|}{$P=0.02$} & \multicolumn{2}{|c|}{$P=0.03$} \\
\hline Summer & 452 & $26 \cdot 4$ & 12 & $2 \cdot 7$ & 22 & 4.9 & 28 & $6 \cdot 2$ & 7 & $2 \cdot c$ \\
\hline Autumn & 397 & $23 \cdot 2$ & 21 & $5 \cdot 3$ & 16 & 4.0 & 46 & 11.6 & 4 & $0 . \varepsilon$ \\
\hline Winter & 510 & 29.8 & 19 & 3.7 & 20 & 3.9 & 53 & 10.4 & 6 & 1.2 \\
\hline Spring & 351 & 20.5 & 20 & $5 \cdot 7$ & 15 & 4.3 & 43 & $12 \cdot 3$ & 15 & $3 \cdot 6$ \\
\hline Total 25 -hydroxyvitamin D at 15 weeks of gestation $(\mathrm{nmol} / \mathrm{l})$ & \multirow{2}{*}{\multicolumn{2}{|c|}{$72 \cdot 9$}} & \multicolumn{2}{|c|}{$P=0.1$} & \multicolumn{2}{|c|}{$P=0.7$} & \multicolumn{2}{|c|}{$P=0.2$} & \multicolumn{2}{|c|}{$P=0.03$} \\
\hline Mean & & & & & & & & & & \\
\hline SD & & & & & & & & & & \\
\hline Total 25-hydroxyvitamin D level at 15 weeks of gestation & & & & & & & & & & \\
\hline$<25 \mathrm{nmol} / \mathrm{l}$ & 76 & & $<5$ & & $<5$ & & 12 & $15 \cdot 8$ & $<5$ & \\
\hline $25-49.9 \mathrm{nmol} / \mathrm{l}$ & 292 & $17 \cdot 1$ & 15 & $5 \cdot 1$ & 14 & 4.8 & 35 & $12 \cdot 0$ & 8 & $2 \cdot 7$ \\
\hline $50-74.9 \mathrm{nmol} / \mathrm{l}$ & 508 & 29.7 & 24 & 4.7 & 16 & 3.1 & 43 & 8.5 & 14 & 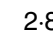 \\
\hline$>75 \mathrm{nmol} / \mathrm{l}$ & 834 & 48.8 & 29 & 3.4 & 39 & 4.7 & 80 & $9 \cdot 6$ & 9 & 1.1 \\
\hline
\end{tabular}

sPTB, spontaneous preterm birth; SGA, small for gestational age; GDM, gestational diabetes mellitus. 
In the SCOPE cohort, there are limited proportions of women with vitamin D concentrations $<25 \mathrm{nmol} / 1$ (4.4\%). We believe that this has not significantly altered the association of other outcomes, given that previous studies investigating the relationship between 25-hydroxyvitmain D concentration and pregnancy outcomes have reported both positive and negative associations in populations with high and low prevalence of vitamin D deficiency (summarised in Table 3).

In some vitamin D-replete populations of pregnant women, significant associations with $\mathrm{GDM}^{(26)}$ and $\mathrm{SGA}^{(27)}$ have been found. In a similarly replete population, investigators found no association between vitamin D status and pregnancy outcomes including pre-eclampsia, SGA, preterm birth and $\mathrm{GDM}^{(20)}$. However, in vitamin D-deficient populations, no association between 25 -hydroxyvitamin $\mathrm{D}$ concentration and $\mathrm{SGA}^{(30)}$ and $\mathrm{GDM}^{(22)}$ was found (Table 3).

The main limitation to our study was the comparatively low prevalence of late pregnancy disorders, particularly GDM; this was due to the purpose for which SCOPE was established - that is, to investigate predictors of late pregnancy disorders in early pregnancy in low-risk, nulliparous women ${ }^{(12)}$. We did not have data on sunlight exposure - a factor that contributes most to 25-hydroxyvitamin D concentration. Given the lack of an association between vitamin $\mathrm{D}$ and pregnancy outcomes in this study, we do not believe these data would have altered the outcome.

Our findings are supported by a recent meta-analysis on trials of vitamin D supplementation in pregnancy and pregnancy outcomes $^{(31)}$. There was no difference in the incidence of pre-eclampsia, preterm birth, low birth weight and GDM with vitamin D supplementation in pregnancy. However, a recently updated Cochrane systematic review on vitamin D supplementation in pregnancy found a reduced incidence of preeclampsia, preterm birth and low birth weight $(<2500 \mathrm{~g})^{(32)}$. These results were sensitive to the inclusion, or not, of trials using low-dose vitamin D supplementation. This highlights the need for pregnancy outcomes to be evaluated in ongoing, randomised trials of vitamin D supplementation in pregnancy.

There may be a number of reasons for the inconsistency in the literature. The aetiology of pre-eclampsia, preterm birth, SGA and GDM are not fully understood and is likely to be multifactorial. Vitamin D deficiency maybe an important predictor in some populations and not in others. For example, because vitamin $\mathrm{D}$ increases intestinal absorption of $\mathrm{Ca}$, in a population where dietary calcium is particularly low, vitamin D status maybe an important predictor for developing pre-eclampsia but not when dietary $\mathrm{Ca}$ is adequate ${ }^{(30)}$. Genetic polymorphisms of the vitamin $\mathrm{D}$ nuclear receptor and vitamin $\mathrm{D}$ binding protein have been associated with a wide variety of conditions ${ }^{(33,34)}$, and may explain why a study in the USA found that vitamin D deficiency $(<37.5 \mathrm{nmol} / \mathrm{l})$ was a risk factor for SGA in white women (adjusted OR $7.5 ; 95 \%$ CI 1.8, 31.9) but not in black women (adjusted OR 1.5; $95 \%$ CI 0.6, 3.5) ${ }^{(28)}$. This study found a non-linear association between 25 -hydroxyvitamin D concentration and SGA, with an increased risk of SGA with concentrations $>75 \mathrm{nmol} / 1$ (OR 2.2; $95 \%$ CI $1 \cdot 2,3 \cdot 8$ ).

Vitamin D may be a surrogate marker for other causative factors that vary between populations, particularly those with 
seasonal variation. We found a significant variation in GDM, pre-eclampsia and SGA with season that was not explained by 25-hydroxyvitamin D concentration. Factors that vary with season may be population specific, especially dietary patterns. A study on pregnant women in New Zealand found significant variations in macronutrient and micronutrient intakes according to season, including lower dietary $\mathrm{Ca}$ consumption in winter and summer ${ }^{(35)}$. A recent study has demonstrated seasonal variation in gene expression in human lymphocytes and adipocytes ${ }^{(36)}$. These cell types play important roles in insulin sensitivity and inflammation. Although it is not yet known how cell function is affected, these variations in gene expression may be relevant to GDM and pre-eclampsia risk. Others have found a significant variation in the prevalence of pre-eclampsia with season $^{(37)}$. In our data, the association of season and GDM remains significant after adjustment for BMI and ethnicity, consistent with greatest risk if early pregnancy occurs during the winter and early spring, when 25-hydroxyvitamin D concentrations are lowest.

In this vitamin D-replete pregnancy cohort, 25-hydroxyvitamin D concentration did not predict pregnancy outcomes including pre-eclampsia, SGA, spontaneous preterm birth and GDM when adjustments were made for confounders. Season was identified as a significant predictor for GDM. This does not exclude a potential contribution of vitamin D to GDM risk, but also raises the possibility of other pertinent seasonal factors.

\section{Acknowledgements}

The authors acknowledge Rennae Taylor (manager), Auckland SCOPE biobank, Department of Obstetrics and Gynaecology, The University of Auckland, and Associate Professor Timothy Kenealy, School of Medicine, The University of Auckland.

Funding was provided by Gravida via Uniservices (grant no. 33015.001), a Centre of Research Excellence of the NZ Government Tertiary Education Commission.

P. N. B. and L. C. K. conceived the study, provided supervision and reviewed the manuscript. V. T. B. analysed samples and the data and drafted the manuscript. E. B. T. assisted with sample analysis and reviewed the manuscript. D. M. assisted with sample analysis. M. B. J. assisted with data analysis and reviewed the manuscript. L. M. E. M. provided the samples and reviewed the manuscript.

The authors have no conflicts of interest to declare.

\section{References}

1. Say L, Chou D, Gemmill A, et al. (2014) Global causes of maternal death: a WHO systematic analysis. Lancet Glob Health 2, e323-e333.

2. You D, Hug L, Ejdemyr S, et al. (2015) Levels and Trends in Child Mortality. New York: WHO.

3. Morisaki N, Esplin MS, Varner MW, et al. (2013) Declines in birth weight and fetal growth independent of gestational length. Obstet Gynecol 121, 51-58.

4. Abitbol CL \& Rodriguez MM (2012) The long-term renal and cardiovascular consequences of prematurity. Nat Rev Nephrol 8, 265-274. 
5. Lawrence JM, Contreras R, Chen W, et al. (2008) Trends in the prevalence of preexisting diabetes and gestational diabetes mellitus among a racially/ethnically diverse population of pregnant women, 1999-2005. Diabetes Care 31, 899-904.

6. Whincup PH, Kaye SJ, Owen CG, et al. (2008) Birth weight and risk of type 2 diabetes: a systematic review. JAMA $\mathbf{3 0 0}$, 2886-2897.

7. Gunderson EP, Chiang V, Pletcher MJ, et al. (2014) History of gestational diabetes mellitus and future risk of atherosclerosis in mid-life: the coronary artery risk development in young adults study. J Am Heart Assoc 3, e000490.

8. Sabour H, Hossein-Nezhad A, Maghbooli Z, et al. (2006) Relationship between pregnancy outcomes and maternal vitamin D and calcium intake: a cross-sectional study. Gynaecol Endocrinol 22, 585-589.

9. Aghajafari F, Nagulesapillai T, Ronksley PE, et al. (2013) Association between maternal serum 25-hydroxyvitamin D level and pregnancy and neonatal outcomes: systematic review and meta-analysis of observational studies. $\mathrm{Br}$ Med J 346, f1169.

10. Zehnder D, Evans KN, Kilby MD, et al. (2002) The ontogeny of 25-hydroxyvitamin $\mathrm{D}_{3} 1 \alpha$-hydroxylase expression in human placenta and decidua. Am J Pathol 161, 105-114.

11. Pijnenborg R, Bland JM, Robertson WB, et al. (1983) Uteroplacental arterial changes related to interstitial trophoblast migration in early human pregnancy. Placenta 4, 397-413.

12. Chappell LC, Seed PT, Myers J, et al. (2013) Exploration and confirmation of factors associated with uncomplicated pregnancy in nulliparous women: prospective cohort study. BMJ 347, f6398.

13. Davis P, McLeod K, Ransom M, et al. (1997) The New Zealand Socio-economic Index of Occupational Status (NZSEI), Statistics New Zealand Research Report. Wellington, New Zealand: Statistics New Zealand.

14. Brown M, Lindheimer M, de Swiet M, et al. (2001) The classification, diagnosis and management of the hypertensive disorders of pregnancy: statement from the International Society for the Study of Hypertension in Pregnancy. Pregnancy Hypertens 20, IX-XIV.

15. North RA, McCowan LME, Dekker GA, et al. (2011) Clinical risk prediction for pre-eclampsia in nulliparous women: development of model in international prospective cohort. BMJ 342, d1875.

16. McCowan L \& Stewart AW (2004) Term birthweight centiles for babies from New Zealand's main ethnic groups. Aust $N Z J$ Obstet Gynaecol 44, 432-435.

17. Carter GD, Berry JL, Gunter E, et al. (2010) Proficiency testing of 25-hydroxyvitamin D (25-OHD) assays. J Steroid Biochem Mol Biol 121, 176-179.

18. Ross AC, Taylor CL, Yaktine AL, et al. (2011) Dietary Reference Intakes for Calcium and Vitamin D. Washington, DC: The National Academies Press.

19. Holick M, Binkley N, HA B-F, et al. (2011) Evaluation, treatment, and prevention of vitamin D deficiency: an Endocrine Society Clinical Practice Guideline. J Clin Endocrinol Metab 96, 1911-1930.

20. Flood-Nichols SK, Tinnemore D, Huang RR, et al. (2015) Vitamin D deficiency in early pregnancy. PLOS ONE 10, $\mathrm{e} 0123763$.

21. Song SJ, Zhou L, Si S, et al. (2013) The high prevalence of vitamin $\mathrm{D}$ deficiency and its related maternal factors in pregnant women in Beijing. PLOS ONE 8, e85081.
22. Park S, Yoon H-K, Ryu H-M, et al. (2014) Maternal vitamin D deficiency in early pregnancy is not associated with gestational diabetes mellitus development or pregnancy outcomes in Korean pregnant women in a prospective study. J Nutr Sci Vitaminol 60, 269-275.

23. Nowson CA, McGrath JJ, Ebeling PR, et al. (2012) Vitamin D and health in adults in Australia and New Zealand: a position statement. Med J Aust 196, 686-687.

24. Women's Health Auckland District Health Board (2008) National women's annual clinical report. Auckland: Auckland District Health Board.

25. Women's Health Auckland District Health Board (2014) Women's health annual clinical report. Auckland: Auckland District Health Board.

26. Lacroix M, Battista MC, Doyon M, et al. (2014) Lower vitamin $\mathrm{D}$ levels at first trimester are associated with higher risk of developing gestational diabetes mellitus. Acta Diabetol 51, 609-616.

27. Schneuer FJ, Roberts CL, Guilbert C, et al. (2014) Effects of maternal serum 25-hydroxyvitamin D concentrations in the first trimester on subsequent pregnancy outcomes in an Australian population. Am J Clin Nutr 99, 287-295.

28. Bodnar LM, Catov JM, Zmuda JM, et al. (2010) Maternal serum 25-hydroxyvitamin D concentrations are associated with small-for-gestational age births in white women. J Nutr $\mathbf{1 4 0}$, 999-1006.

29. Akcakus M, Koklu E, Budak N, et al. (2006) The relationship between birthweight, 25-hydroxyvitamin D concentrations and bone mineral status in neonates. Ann Trop Paediatr 26, 267-275.

30. Hoffmeyr GJ, Lawrie TA, Atallah AN, et al. (2014) Calcium supplementation during pregnancy for preventing hypertensive disorders and related problems. Cochrane Database Syst Rev, issue 6, CD001059.

31. Pérez-López FR, Pasupuleti V, Mezones-Holguin E, et al. (2015) Effect of vitamin D supplementation during pregnancy on maternal and neonatal outcomes: a systematic review and meta-analysis of randomized controlled trials. Fertil Steril 103, 1278-1288.e4.

32. De-Regil LM, Palacios C, Lombardo LK, et al. (2016) Vitamin D supplementation for women during pregnancy. Cochrane Database Syst Rev, issue 1, CD008873.

33. Gnagnarella P, Pasquali E, Serrano D, et al. (2014) Vitamin D receptor polymorphism FokI and cancer risk: a comprehensive meta-analysis. Carcinogenesis 35, 1913-1919.

34. Koplin JJ, Suaini NHA, Vuillermin P, et al. (2016) Polymorphisms affecting vitamin D-binding protein modify the relationship between serum vitamin $\mathrm{D}(25[\mathrm{OH}] \mathrm{D} 3)$ and food allergy. J Allergy Clin Immunol 137, 500-506.e4.

35. Watson PE \& McDonald BW (2007) Seasonal variation of nutrient intake in pregnancy: effects on infant measures and possible influence on diseases related to season of birth. Eur J Clin Nutr 61, 1271-1280.

36. Dopico XC, Evangelou M, Ferreira RC, et al. (2015) Widespread seasonal gene expression reveals annual differences in human immunity and physiology. Nat Commun 6, 7000.

37. TePoel MRW, Saftlas AF \& Wallis AB (2011) Association of seasonality with hypertension in pregnancy: a systematic review. J Reprod Immunol 89, 140-152.

38. Kim C, Newton KM \& Knopp RH (2002) Gestational diabetes and the incidence of type 2 diabetes: a systematic review. Diabetes Care 25, 1862-1868. 